Research Paper

\title{
SYT12 is a novel oncogene that promotes thyroid carcinoma progression and metastasis
}

Lingli Jin\#, Danni Zheng\#, Danxiang Chen, Erjie Xia, Yaoyao Guan, Jialiang Wen, Adheesh Bhandari ${ }^{\bowtie}$ and Ouchen Wang ${ }^{\bowtie}$

Department of Breast Surgery, The First Affiliated Hospital of Wenzhou Medical University, Wenzhou, Zhejiang, PR China.

\#These authors contributed equally to this work.

$\triangle$ Corresponding authors: Prof. Dr. Adheesh Bhandari, Department of Breast Surgery, The First Affiliated Hospital of Wenzhou Medical University, Wenzhou, Zhejiang, People's Republic of China. Contact no.: +86 5775557 8527; E-mail: adheesh2003@hotmail.com; Prof. Dr. Ouchen Wang, Department of Breast Surgery, The First Affiliated Hospital of Wenzhou Medical University, Wenzhou, Zhejiang, People's Republic of China. Contact no.: +86 577 5557 8527; E-mail: woc863@hotmail.com.

(C) The author(s). This is an open access article distributed under the terms of the Creative Commons Attribution License (https://creativecommons.org/licenses/by/4.0/). See http://ivyspring.com/terms for full terms and conditions.

Received: 2021.05.10; Accepted: 2021.09.01; Published: 2021.09.27

\begin{abstract}
Background: Thyroid malignancy is the most frequent endocrine malignant tumor whose incidence is still increasing. Mechanisms genomic variations play a major part in the pathogenesis of many types of malignancy. Synaptotagmin 12 (SYT12) is a member gene of the synaptotagmins family and SYT12's variants were shown to be associated with some malignancies. Nevertheless, SYT12's specific function and probable clinical value in papillary cancer were still unknown.

Methods: We conducted complete genome sequence of 39 pairs PTC malignant neoplasm and matched non-neoplastic tissues. We found that SYT12 was significantly overexpressed in thyroid malignancy. Next, we investigated the expression level of SYT12 and the relation between clinical information and SYT12 expression in thyroid cancer in the Cancer Genome Atlas (TCGA). QRt-PCR of else 40 pairs local verified cohort was performed to confirm the sequencing data and TCGA cohort. Then, we used small interfering RNA (si-RNA) to knock down the expression of SYT1 2 in PTC cells. Finally, proliferation, cell colony formation, migration, invasion, and apoptosis assays were done to demonstrate the function of SYT12.

Results: SYT12 is significantly overexpressed and higher expression of SYT12 upsurges the risk of lymph node metastatic and incidence rate of primary neoplasm multivariate focus type and classical histological type for PTC patients in TCGA cohort. In vitro experiments, the results of functional assays presented that knock-down of SYT12 inhibited the cell proliferation, cell colony formation, trans-well migration, and trans-well invasion and promoted cell apoptotic in PTC cell lines.
\end{abstract}

Conclusion: SYT1 2 was a novel oncogene that promotes thyroid carcinoma progression and metastasis potential and a potential biomarker for diagnosis and treatment in PTC.

Key words: PTC, SYT12, biomarker, LNM, primary neoplasm focus type, histological type

\section{Introduction}

Thyroid carcinoma is the most frequent endocrine malignant tumor worldwide. According to the United States cancer statistics, 52890 lately assessed identified cases and 2180 expected deaths in 2020 [1]. Except for morbidity number of thyroid malignancies gradually increase, the average age of morbidity crowd also become younger. Thyroid cancer cases upsurge by about 3\% yearly amongst those whose age were 20-39 and 4\% amongst those whose age were 20-39 [2]. Thyroid cancer was divided into many types. And among those papillary thyroid cancer (PTC) is the most frequent generic pathological pattern of thyroid malignant tumor, making up almost $80 \%$ of thyroid cancers. In contrast to the rising occurrence of thyroid malignancy, the mortality rate of thyroid cancers has remained stable the same 
period despite [3-6]. And the estimated overdiagnosis rates for thyroid cancers range from $50 \%$ to $90 \%$ of newly diagnosed cases in women, under the influence of different regions and health care environment [4]. Hence, an appropriate and precise diagnosis is essential to reducing the overtreatment of nonfatal thyroid cancers.

With the increasing knowledge of the molecular, it was recognized that mechanisms genomic variations play a major role in the pathogenesis of many types of malignancies, involving point mutations in chromosomal rearrangements and proto-oncogenes. MAPK activation could accelerate PTC initiation and progression and the mutations of BRAF V600E could activate MAPK. The activation of PI3K/AKT passage is also deeded be significant for initiation of thyroid cancer. he genetic mutation of RAS, PI3CA, AKT and PTEN all could stimulate this pathway [7]. Despite the significant progress made in genetic research, the mechanisms underlying PTC tumorigenesis remain unclear and further research needs to be done to discover a more precise forecast modal.

Synaptotagmin 12 (SYT12) is a member of the synaptotagmin (SYT) family. As we all know, the synaptotagmin family regulated the release of neurotransmitters according accommodating the fusion of calcium-dependent synaptic vesicle membrane proteins. There is an N-terminal transmembrane region and a tandem $\mathrm{C}$-terminal $\mathrm{C} 2$ domains in some SYTs [8]. C2 domains serve as a connector connecting Ca2+. SYT1-3, 5-7 and 9 own C2 domains while SYT4, 8, and 11-16 do not [9].

In recent years, variants of the human SYT12 were shown to be associated with some cancers [10, 11]. Liu et al reported that SYT12 acted as a possible oncogene by activating the PI3K-AKT-mTOR signal channel in lung adenocarcinoma [11]. Keitaro et al also found that SYT12 played a crucial part in oral carcinoma and might be an innovative therapeutic target [10]. And Jonklaas et al. showed that SYT12 may contribute to predicting PTC results in a prospective cohort, but they did not verify the specific biological functions by biology empirical study [12]. In brief, the function of the SYT12 gene play in the beginning and progression of thyroid malignancies is still keeping mysterious. Therefore, a further research of the biological purposes about the human being SYT12 is significant to comprehend PTC tumorigenesis and evolution. The present investigation expected to discover the relationship amongst the expression level of human SYT12 and clinical descriptions, and observe the SYT12's biological property by utilizing small interfering RNA (si-RNA) in PTC cells.

\section{Materials and Methods}

Patients and Thyroid Tissue samples collection

We acquired 79 (39 pairs PTC malignant neoplasm and matched non-neoplastic tissues genetic for genetic sequencing of incomplete research project; 40 pairs PTC malignant neoplasm and matched non-neoplastic tissues genetic for real-time reverse transcription-quantitative polymerase chain reaction) pairs PTC tumor and nearby non-neoplastic thyroid tissues from 79 PTC patients who were diagnosed by two pathologists at least. The inclusion criteria of those 79 PTC patients were as described below: (1) Having surgery in Department of Thyroid and Breast Surgery, The First Affiliated Hospital of Wenzhou Medical University, Wenzhou, Zhejiang, People's Republic of China; (2) one lymph node was examined at least (3) having complete clinical baseline features; (4) no preoperative radiotherapy; (5) no another malignant tumor was diagnosed before; (6) site recode according to the Third Edition of International Classification of Disease for Oncology (ICD-O-3): C73.9 Thyroid gland; (7) morphological recode according to ICD-O-3:8340/3 Papillary carcinoma, follicular variant; 8341/3 Papillary microcarcinoma; 8342/3 Papillary carcinoma, oxyphilic cell; 8343/3 Papillary carcinoma, encapsulated; 8344/3 Papillary carcinoma, columnar cell, and; (8) the stage according to the $7^{\text {th }}$ edition AJCC staging system: I-IV.

After resection, samples were put in liquid nitrogen as quickly as possible. If the samples were not used for RNA extraction immediately, they would be stored in $-80{ }^{\circ} \mathrm{C}$ refrigerator. There was another cohort in our study and it was gained from the Cancer Genome Atlas Database (TCGA) database. In complete, we elected 502 PTCs and 58 non-neoplastic thyroid tissues with whole sequence data and complete clinical features. Each procedure was recorded with the ethical standards approval of the Ethics Committee of the First Affiliated Hospital of Wenzhou Medical University.

\section{RNA extraction, reverse transcription and real-time reverse transcription-quantitative polymerase chain reaction (RT-qPCR)}

The genetic code data of 39 paired PTC samples and matched adjacent non-neoplastic thyroid samples were originated from another incomplete research project. We isolated the RNA from fresh tissues and cells by TRIzol (Thermo Fisher Scientific, Waltham, MA, USA). We inspected the quantity and quality of isolated RNA by A260/A280 ratio and spectrophotometric value. It was deemed as most efficient that $1 \mu \mathrm{g}$ RNA per $20 \mu \mathrm{l}$ system reacting contained. And a SYBR Premix Ex Taq II kit (RR820A, TaKaRa, 
Dalian, China) was used for Real-time reverse transcription-quantitative polymerase chain reaction (qRt-PCR) on an applied Biosystems 7500 Real-Time PCR System. The mRNA expression comparing with GAPDH expression was calculated by the $2-\Delta \Delta \mathrm{Ct}$ equation. All procedures were performed by standard instructions. The sequences of SYT12 primer were bought from Sangon Biotech (Shanghai, China), as described below: SYT12,5'-GCAACACCTTTGGGCA GGAC-3' (forward) and 5'-GTGTGG GAGGCAG TGTCGTA -3' (reverse). Each assay was performed in triplicate and the steps were performed by standard description.

\section{Cell lines and cell culture}

Professor Mingzhao Xing of Johns Hopkins University School of Medicine (Baltimore, MD, USA) provided human PTC cell lines (TPC, KTC and BCPAP) which we needed. The Cell Bank of the Shanghai Chinese Academy of Sciences (Shanghai, China) offered the normal thyroid cell line (HTORI3) of human. All cells were cultivated in RPMI 1640 (Invitrogen; Thermo Fisher Scientific, Inc., Waltham, MA, USA) supplemented with 1X DMEM nonessential amino acids, $10 \%$ fetal bovine serum (FBS; Invitrogen; Thermo Fisher Scientific, Inc.) and 1X sodium pyruvate. And the nutrient solution containing cells was kept in incubator with $\mathrm{CO} 2$ concentration in $5 \%$ at $37^{\circ} \mathrm{C}$.

\section{siRNA Transfection}

We knock-down the gene of SYT12 by small interfering RNA (siRNA) which was purchased by Gene Pharma (Shanghai, China). The siRNA sequences of SYT12 used in the present study were as follows:

siRNA1, forward 5'-GCAGAAUACCAUCUGA GCGTT-3' and reverse 5'-CGCUCAGAUGGUAUU CUGCTT-3'; siRNA2, forward 5'-UCAUCUGGACCA ACGACAATT-3' and reverse 5'-UUGUCGUUGGU CCAGAUGATT-3'; siRNA3, forward 5'-CCAUCUU CUUUGAUGAGAATT-3' and reverse 5'-UUCUCA UCAAAGAAGAUGGTT-3'.

Those three siRNAs were all targeting to knock down the expression of SYT12, and different siRNA sequences had different knockout efficiency. Si-NC was offered by Gene Pharma as blank control when we bought the siRNAs. PTC cells were seeded into six-well plates (TPC $7 \times 10^{4}$ cells/well; KTC $8 \times 10^{4}$ cells/well) and cultivated for 24 hours before transfection. The expression of SYT12 was reduced by small interfering RNA (si-RNA) which was delivered to cells by a membrane destructive agent named RNAiMAX (Invitrogen, Grand Island, NY, USA) (si-RNA: RNAiMAX $=7.5 \mu \mathrm{l}: 3 \mu \mathrm{l}$ ) according to the standard description. After 48 hours' of cultivating, cells were collected for following tests.

\section{Cell proliferation assay}

Cell-counting kit 8 reagent (CCK-8, Beyotime, Biotechnology, Shanghai, China) was used to observe the proliferation ability of PTC cells. TPC and KTC cells which had been exposed in SYT12-siRNAs or NC-siRNA for 48 hours were seeded onto 96-well plates (1000 cells/plate). Then, the CCK-8 (10 $\mu \mathrm{l} /$ well) (Beyotime Biotechnology, Shanghai, China) was added to wells, and then the cells were incubated with at $37^{\circ} \mathrm{C}$ for 3 hours. In the next four days, $450 \mathrm{~nm}$ absorbance was measured to drawn proliferation curves by spectrophotometer (DS-11 FX; DeNovix, Wilmington, USA). The experiment was repeated three times.

\section{Colony formation assay}

After 48 hours transfection, the thyroid cancer TPC $\left(1 \times 10^{3}\right.$ cells $)$ and KTC cells $\left(2 \times 10^{3}\right.$ cells $)$ were plated into a 6-well plate. There were more than 50 cells in a colony formation, 8 days later. We fixed the cells by $4 \%$ paraformaldehyde for 30 minutes. Then, $0.1 \%$ crystal violet solution was used to stain the cells for 30 minutes. Those colonies which were more than 50 cells in a colony formation were counted and images were captured by digital camera. This was repeated three times. Altogether, experiments were achieved in triplicate.

\section{Migration and invasion assays}

Transwell chambers (\#3422, Corning, NY, USA) were used to measure the migration ability of PTC cells. TPC and KTC cells $\left(3 \times 10^{4}\right.$ cells/well $)$ which had been transfected for 48 hours were seeded into the upper chamber in $300 \mu \mathrm{l}$ serum-free nutrient solution while the lower chamber contained $600 \mu \mathrm{l}$ medium supplemented with 10\% FBS. 22 hours later, the cells were fixed by $4 \%$ paraformaldehyde for 30 minutes. Then, $0.1 \%$ crystal violet solution was used to stain the cells for 30 minutes. We captured the pictures by microscope under the magnifying power of $10 \times 40$ for further analysis. BioCoat Matrigel Invasion Chambers (\#354480, Corning Biocoat, USA) were used to exam the invasion ability and the procedures were similar to migration assay. The experiment was repeated three times.

\section{Apoptosis assays}

Cell apoptosis ability was detected by Annexin V-fluorescein isothiocyanate (FITC) apoptosis kit (\#556547; Becton, Dickinson and Company, Franklin Lakes, NJ, USA) with specification of manufacturer. After 48 hours transfection, cells were gathered and washed 3 times by phosphate-buffered saline (PBS). 
Then, we resuspended the cells into $1 \times$ binding buffer $\left(1 \times 10^{6}\right.$ cells $\left./ \mathrm{ml}\right)$. Next, cells were stained with Annexin V-FITC for 15 minutes and propidium iodide (PI) for 5 minutes in the dark before examined by flow cytometry (BD Biosciences Accuri C6; Becton, Dickinson and Company). And the results were analyzed by Flowjo software (Flowjo, Ashland, OR, USA). The experiment was repeated three times.

\section{Statistical analysis}

All assays were repeated three times and data was shown as the mean \pm SD. SPSS 25.0 software and GraphPad Prism 7.0 were the analytical software used to analyze the data. Student's t-test was used to evaluate two group comparisons and one-way ANOVA was used to analyze multiple group comparisons. $\mathrm{P}<0.05$ was considered to be significant statistical difference.

\section{Results}

\section{SYT1 2 is obviously overexpressed in PTC}

To make an investigation of the function of SYT12 in PTC, we initially detected the expression level of SYT12 in 40 pairs tumor samples and nearby normal thyroid tissue via RT-qPCR. After analyzing this data, we identified that SYT12 expression in tumors was significantly upregulated. To describe the

A

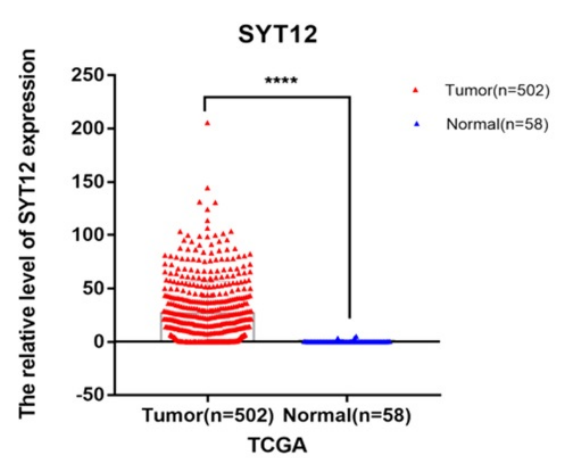

C

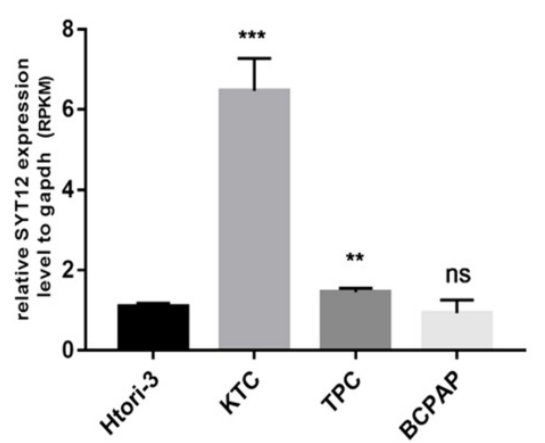

difference in our verified cohort, a heat map was made (40 pairs tumor tissues and matched noncancerous tissues, $\Delta d=-66.36 \pm 58.66$, paired t-test, $\mathrm{P}<$ 0.0001 ) (Figure 1B). This result is consistent with the TCGA data (Tumor tissue $26.99 \pm 1.321, \mathrm{n}=502$; Normal tissue $0.3156 \pm 0.1181, \mathrm{n}=58$; unpaired $\mathrm{t}$-test $\mathrm{P}$ $<0.0001$ ) (Figure 1A). Next, we test the mRNA expression of SYT12 in different thyroid cancer cell lines, the result is also the same as collected surgical tissue samples and TCGA cohort. The mRNA expression level of SYT12 in different PTC cell lines were obviously higher than non-neoplastic thyroid cell line, just like the trend of tissues (compared with HTORI-3, KTC, $\mathrm{P}=0.0003$; TPC, $\mathrm{P}=0.0082$; $\mathrm{BCPAP}$, $\mathrm{P}=0.4684$ ) (Figure 1C).

\section{Connection between the expression of SYT1 2 and clinical factors}

To examine the part of SYT12 taking in the occurrence and development of PTC, we analyze the association between the expression level of SYT12 and clinical factors. And the whole patients were segmented into 2 sets (high expression group and low expression group), according to median value. We found that the histological type $(\mathrm{P}<0.001)$, primary neoplasm focus type $(\mathrm{P}=0.044)$, tumor stage $(\mathrm{P}=0.001)$, lymph node metastasis $(\mathrm{P}<0.001)$ and the disease stage $(\mathrm{P}<0.001)$ were obviously different in the 2 sets

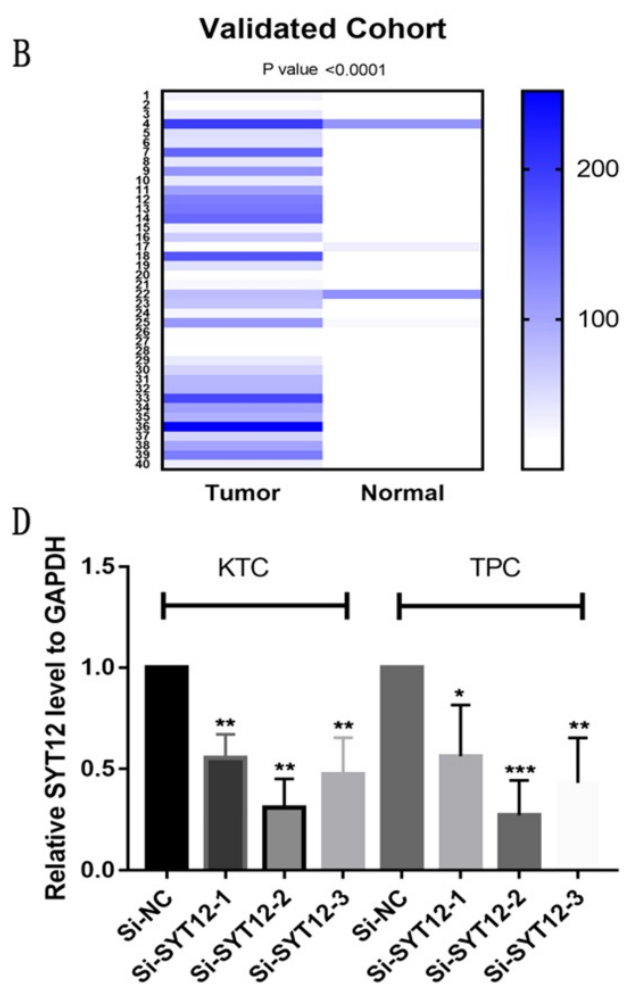

Figure 1. SYT1 2 expression in thyroid cancer in the TCGA cohort. (A) The mRNA expression level of SYT12 in PTC in TCGA cohort. (B) A hot map that describes the SYT12 expression examined by RT-qPCR in 40 paired thyroid cancer tissues and adjacent noncancerous tissues. (C) The relative expression of SYT12 in PTC cell lines. SYT12 was upregulated in two PTC cell lines (TPC and KTC) compared to normal thyroid cell line HTORI-3. (D) SYT12 expression levels of si-SYT12 groups and si-NC in the two PTC cell lines. $* \mathrm{P}<0.05, * * \mathrm{p}<0.01, * * * \mathrm{p}<0.001, * * * * \mathrm{p}<0.0001$. 
(Table 1) in TCGA cohort. But in the local cohort, we did not find the similar consequence as demonstrated in the TCGA cohort. 40 thyroid cancer tissues were analyzed in the local cohort (Table 2), there is no significant difference about primary neoplasm focus type $(\mathrm{P}=0.191)$, tumor stage $(\mathrm{P}=0.516)$, lymph node metastasis $(\mathrm{P}=0.324)$ and the disease stage $(\mathrm{P}=0.101)$. Probably because the volumes of local validated cohort were too small to draw a credible result.

Table 1. Association between the expression of SYT12 and clinicopathological factors in the TCGA cohort

\begin{tabular}{|c|c|c|c|c|c|}
\hline $\begin{array}{l}\text { Clinicopathologic } \\
\text { factors }\end{array}$ & Patients & $\begin{array}{l}\text { High } \\
\text { expression (\%) }\end{array}$ & $\begin{array}{l}\text { Low } \\
\text { expression (\%) }\end{array}$ & $x^{2}$ & p-value \\
\hline \multicolumn{6}{|l|}{ Gender } \\
\hline Female & 128 & $64(50)$ & $64(50)$ & & \\
\hline Male & 360 & $182(50.6)$ & $178(49.4)$ & 0.012 & 0.914 \\
\hline \multicolumn{6}{|l|}{ Age(years) } \\
\hline$<45$ & 229 & $111(48.5)$ & $118(51.5)$ & & \\
\hline$\geq 45$ & 259 & $135(52.1)$ & $124(47.9)$ & 0.648 & 0.421 \\
\hline \multicolumn{6}{|l|}{ Histological type } \\
\hline Classical & 346 & $203(58.7)$ & $143(41.3)$ & & \\
\hline Other types & 142 & $43(30.3)$ & $99(69.7)$ & 32.459 & $<0.001^{* * *}$ \\
\hline \multicolumn{6}{|c|}{ Primary neoplasm focus type } \\
\hline Unifocal & 264 & $122(46.2)$ & $142(53.8)$ & & \\
\hline Multifocal & 224 & $124(55.4)$ & $100(44.6)$ & 4.054 & $0.044^{*}$ \\
\hline \multicolumn{6}{|l|}{ Tumor stage } \\
\hline $\mathrm{I}+\mathrm{II}$ & 296 & $131(44.3)$ & $165(55.7)$ & & \\
\hline $\mathrm{III}+\mathrm{IV}$ & 192 & $115(59.9)$ & $77(40.1)$ & 11.394 & $0.001^{* *}$ \\
\hline \multicolumn{6}{|c|}{ Lymph node metastasis } \\
\hline No & 272 & $104(38.2)$ & $168(61.8)$ & & \\
\hline Yes & 216 & $142(65.7)$ & $74(34.3)$ & 36.436 & $<0.001^{* * *}$ \\
\hline \multicolumn{6}{|c|}{ Disease stage (AJCC7) } \\
\hline $\mathrm{I}+\mathrm{II}$ & 323 & $143(44.3)$ & $180(55.7)$ & & \\
\hline III+IV & 165 & $103(62.4)$ & $62(37.6)$ & 14.394 & $<0.001^{* * *}$ \\
\hline \multicolumn{6}{|l|}{ Distant metastasis } \\
\hline No & 479 & $242(50.5)$ & $237(49.5)$ & & \\
\hline Yes & 9 & $4(44.4)$ & $5(55.6)$ & 0.131 & 0.718 \\
\hline \multicolumn{6}{|l|}{ New event } \\
\hline No & 444 & $226(50.9)$ & $218(49.1)$ & & \\
\hline Yes & 44 & $20(45.5)$ & $24(54.5)$ & 0.475 & 0.491 \\
\hline \multicolumn{6}{|l|}{ Status } \\
\hline Alive & 472 & $239(50.6)$ & $233(49.4)$ & & \\
\hline Dead & 16 & $7(43.8)$ & $9(56.2)$ & 0.294 & 0.588 \\
\hline
\end{tabular}

Notes: ${ }^{*}$ p-value $<0.05,{ }^{* *}$ p-value $<0.01,{ }^{* * *}$ p-value $<0.001$;

Abbreviations: SYT12, synaptotagmin 12; AJCC7, American Joint Committee on Cancer $7^{\text {th }}$ edition.
Table 2. Association between the expression of SYT12 and clinicopathological factors in the validated cohort

\begin{tabular}{|c|c|c|c|c|c|}
\hline $\begin{array}{l}\text { Clinicopathologic } \\
\text { factors }\end{array}$ & Patients & $\begin{array}{l}\text { High } \\
\text { expression (\%) }\end{array}$ & $\begin{array}{l}\text { Low } \\
\text { expression (\%) }\end{array}$ & $x^{2}$ & $\mathrm{p}$-value \\
\hline \multicolumn{6}{|l|}{ Gender } \\
\hline Female & 26 & $13(50)$ & $13(50)$ & & \\
\hline Male & 14 & $6(42.9)$ & $8(57.1)$ & 0.186 & 0.666 \\
\hline \multicolumn{6}{|l|}{ Age (years) } \\
\hline$<45$ & 26 & $13(50)$ & $13(50)$ & & \\
\hline$\geq 45$ & 14 & $6(42.9)$ & $8(57.1)$ & 0.186 & 0.666 \\
\hline \multicolumn{6}{|c|}{ Primary neoplasm focus type } \\
\hline Unifocal & 31 & $13(41.9)$ & $18(58.1)$ & & \\
\hline Multifocal & 9 & $6(66.7)$ & $3(33.3)$ & 1.711 & 0.191 \\
\hline \multicolumn{6}{|l|}{ Tumor stage } \\
\hline I+II & 19 & $8(42.1)$ & $11(57.9)$ & & \\
\hline $\mathrm{III}+\mathrm{IV}$ & 21 & $11(52.4)$ & $10(47.6)$ & 0.422 & 0.516 \\
\hline \multicolumn{6}{|c|}{ Lymph node metastasis } \\
\hline No & 18 & $7(38.9)$ & $11(61.1)$ & & \\
\hline Yes & 22 & $12(54.5)$ & $10(45.5)$ & 0.973 & 0.324 \\
\hline \multicolumn{6}{|c|}{ Disease stage (AJCC7) } \\
\hline I+II & 34 & $18(52.9)$ & $16(47.1)$ & & \\
\hline $\mathrm{III}+\mathrm{IV}$ & 6 & $1(16.7)$ & $5(83.3)$ & 2.691 & 0.101 \\
\hline
\end{tabular}

\section{Higher expression of SYT 12 increases the risk of the incidence rate of primary neoplasm multivariate focus type in patients with PTC}

So as to examine the role of SYT12 expression take part in primary neoplasm multivariate focus type, we performed logistic regression. Univariate logistic regression analysis in TCGA cohort shown that the meaningful variables for primary neoplasm multivariate focus type were expression level of SYT12 $(\mathrm{P}=0.047)$, gender $(\mathrm{P}=0.002)$, histological type $(\mathrm{P}=0.002)$ and status $(\mathrm{P}=0.023)$ (Table 3). Multivariate logistic analysis presented that the expression level of SYT12 $(\mathrm{P}=0.008)$, gender $(\mathrm{P}=0.001)$, histological type $(\mathrm{P}=0.005)$ and status $(\mathrm{P}=0.021)$ were the significant variables for primary neoplasm multivariate focus type (Table 3). In short, upregulated expression of SYT12 serves as a risk of primary neoplasm multivariate focus type.

Table 3. Univariate and multivariate logistic regression analysis for the primary neoplasm focus type in the TCGA cohort

\begin{tabular}{|c|c|c|c|c|c|c|}
\hline \multirow[t]{2}{*}{ Clinicopathologic factors } & \multicolumn{3}{|c|}{ Univariate analysis } & \multicolumn{3}{|c|}{ Multivariate analysis } \\
\hline & $\overline{\mathrm{OR}}$ & $95 \% \mathrm{CI}$ & P-value & OR & $95 \% \mathrm{CI}$ & P-value \\
\hline STYT12 Expression (high vs. low) & 1.506 & $1.006-2.253$ & $0.047^{*}$ & 1.681 & $1.145-2.470$ & $0.008^{* *}$ \\
\hline Gender (female vs. male) & 0.499 & $0.324-0.769$ & $0.002^{* *}$ & 0.488 & $0.321-0.744$ & $0.001^{* *}$ \\
\hline Age $(>45$ vs. $<45)$ & 1.143 & $0.682-1.916$ & 0.611 & & - & \\
\hline Histological type (classical vs. others) & 2.007 & $1.291-3.120$ & $0.002^{* *}$ & 0.55 & $0.361-0.838$ & $0.005^{* *}$ \\
\hline Lymph node metastasis (yes vs. no) & 1.467 & $0.943-2.281$ & 0.089 & & - & \\
\hline Tumor stage (III, IV vs. I, II) & 1.031 & $0.651-1.635$ & 0.896 & & - & \\
\hline Disease stage (AJCC7) (yes vs. no) & 1.079 & $0.572-2.035$ & 0.815 & & - & \\
\hline Distant metastasis (yes vs. no) & 0.121 & $0.014-1.041$ & 0.054 & & - & \\
\hline New event (yes vs. no) & 0.793 & $0.400-1.571$ & 0.506 & & - & \\
\hline Status (dead vs. alive) & 0.168 & $0.036-0.785$ & $0.023^{*}$ & 0.169 & $0.037-0.762$ & $0.021^{*}$ \\
\hline
\end{tabular}

Notes: ${ }^{*}$-value $<0.05,{ }^{* *}$ p-value $<0.01,{ }^{* * *}$ p-value $<0.001$

Abbreviations: SYT12, synaptotagmin 12; AJCC7, American Joint Committee on Cancer $7^{\text {th }}$ edition. 
Table 4. Univariate and multivariate logistic regression analysis for the lymph node metastatic risk in the TCGA cohort

\begin{tabular}{|c|c|c|c|c|c|c|}
\hline \multirow[t]{2}{*}{ Clinicopathologic factors } & \multicolumn{3}{|c|}{ Univariate analysis } & \multicolumn{3}{|c|}{ Multivariate analysis } \\
\hline & OR & $95 \% \mathrm{CI}$ & P-value & OR & $95 \% \mathrm{CI}$ & P-value \\
\hline STYT12 Expression (high vs. low) & 2.334 & $1.500-3.631$ & $<0.001^{* * *}$ & 2.328 & $1.514-3.579$ & $<0.001^{* * *}$ \\
\hline Gender (female vs. male) & 0.67 & $0.407-1.104$ & 0.116 & & - & \\
\hline Age $(>45$ vs. $<45)$ & 0.031 & 0.009-0.102 & $<0.001^{* * *}$ & 0.031 & $0.010-0.103$ & $<0.001^{* * *}$ \\
\hline Histological type (classical vs. others) & 2.896 & $1.721-4.874$ & $<0.001^{* * *}$ & 2.645 & $1.615-4.331$ & $<0.001^{* * *}$ \\
\hline Primary neoplasm focus type (Mul vs. Uni) & 1.558 & $0.996-2.436$ & 0.052 & & - & \\
\hline Tumor stage (III,IV vs. I,II) & 0.971 & $0.590-1.598$ & 0.909 & & - & \\
\hline Disease stage(AJCC7) (yes vs. no) & 56.886 & $16.118-200.769$ & $<0.001^{* * *}$ & 60.017 & $17.997-200.145$ & $<0.001^{* * *}$ \\
\hline Distant metastasis (yes vs. no) & 0.756 & 0.164-3.491 & 0.72 & & - & \\
\hline New event (yes vs. no) & 1.592 & $0.771-3.289$ & 0.209 & & - & \\
\hline Status (dead vs. alive) & 0.978 & $0.269-3.563$ & 0.974 & & - & \\
\hline
\end{tabular}

Notes: *p-value $<0.05,{ }^{* *}$ p-value $<0.01,{ }^{* * *}$ p-value $<0.001$;

Abbreviations: SYT12, synaptotagmin 12; AJCC7, American Joint Committee on Cancer $7^{\text {th }}$ edition.

Table 5. Univariate and multivariate logistic regression analysis for the histological type in the TCGA cohort

\begin{tabular}{|c|c|c|c|c|c|c|}
\hline \multirow[t]{2}{*}{ Clinicopathologic factors } & \multicolumn{3}{|c|}{ Univariate analysis } & \multicolumn{3}{|c|}{ Multivariate analysis } \\
\hline & OR & $95 \% \mathrm{CI}$ & P-value & OR & $95 \% \mathrm{CI}$ & P-value \\
\hline STYT12 Expression (high vs. low) & 3.461 & $2.183-5.487$ & $<0.001^{* * *}$ & 3.314 & $2.103-5.224$ & $<0.001^{* * *}$ \\
\hline Gender (female vs. male) & 0.834 & $0.503-1.383$ & 0.483 & & - & \\
\hline Age $(>45$ vs. $<45)$ & 0.806 & $0.461-1.411$ & 0.451 & & - & \\
\hline Lymph node metastasis (yes vs. no) & 2.669 & $1.610-4.425$ & $<0.001^{* * *}$ & 2.773 & $1.726-4.457$ & $<0.001^{* * *}$ \\
\hline Primary neoplasm focus type (Mul vs. Uni) & 0.494 & $0.317-0.769$ & $0.002^{* *}$ & 0.494 & $0.320-0.764$ & $0.002^{* *}$ \\
\hline Tumor stage (III, IV vs. I, II) & 0.544 & $0.312-0.948$ & $0.032^{*}$ & 0.501 & $0.316-0.794$ & $0.003^{* *}$ \\
\hline Disease stage (AJCC7) (yes vs. no) & 0.846 & $0.312-0.948$ & $0.652^{*}$ & & - & \\
\hline Distant metastasis (yes vs. no) & 0.163 & $0.031-0.861$ & $0.033^{*}$ & 0.178 & $0.035-0.917$ & $0.039^{*}$ \\
\hline New event (yes vs. no) & 1.291 & $0.584-2.857$ & 0.528 & & - & \\
\hline Status (dead vs. alive) & 11.792 & $1.350-103.025$ & $0.026^{*}$ & 10.763 & $1.245-93.045$ & $0.031^{*}$ \\
\hline
\end{tabular}

Notes: ${ }^{*}$ p-value $<0.05,{ }^{* *}$ p-value $<0.01,{ }^{* * *}$ p-value $<0.0011$

Abbreviations: SYT12, synaptotagmin 12; AJCC7, American Joint Committee on Cancer $7^{\text {th }}$ edition.

\section{Higher expression of SYT 12 increases the risk of lymph node metastatic in patients with PTC}

To further examine whether the level of SYT12 expression was a major influencing factor of lymph node metastatic, univariate and multivariate logistic regression analysis were done in TCGA cohort. Univariate logistic regression analysis in TCGA cohort confirmed that the crucial variables for the risk of lymph node metastatic were the expression level of SYT12 $(\mathrm{P}<0.001)$, age $(\mathrm{P}<0.001)$, histological type $(\mathrm{P}<0.001)$ and disease stage $(\mathrm{AJCC} 7)(\mathrm{P}<0.001)$ (Table 4). Multivariate logistic analysis displayed that the expression of SYT12 $(\mathrm{P}<0.001)$, age $(\mathrm{P}<0.001)$, histological type $(\mathrm{P}<0.001)$ and disease stage $(\mathrm{AJCC})$ $(\mathrm{P}<0.001)$ impacted the risk of lymph node metastatic (Table 4). That is, the upregulation of SYT12 aggrandized the risk of LNM.

\section{Higher expression of SYT 12 increases the risk of the incidence rate of classical histological type in patients with PTC}

We found the expression level of SYT12 is a significant risk factor for the risk of incidence rate of classical histological type by logistic regression. Univariate logistic regression analysis in TCGA cohort confirmed that the crucial variables for the risk of incidence rate of classical histological type were the expression of SYT12 (P<0.001), lymph node metastasis $(\mathrm{P}<0.001)$, primary neoplasm focus type $(\mathrm{P}=0.002)$, tumor stage $(\mathrm{P}=0.032)$, distant metastasis $(\mathrm{P}=0.033)$ and status $(\mathrm{P}=0.026)$ (Table 5). Multivariate logistic analysis showed the same result that the expression of SYT12 ( $<<0.001)$, lymph node metastasis $(\mathrm{P}<0.001)$, primary neoplasm focus type $(\mathrm{P}=0.002)$, tumor stage $(\mathrm{P}=0.003)$, distant metastasis $(\mathrm{P}=0.031)$ were connected with the risk of the risk of incidence rate of classical histological type. All the above suggested that overexpression of SYT12 increased the risk of incidence rate of classical histological type in PTC.

\section{Knock-down of SYT1 2 inhibited the proliferation of PTC cells}

The SYT12 gene is usually over-expression in PTC, it was assumed that SYT12 plays a significant role in tumorigenesis and progression in thyroid cancer. First, we test the expression of SYT12 in different PTC cell lines by RT-qPCR analysis. We found that this gene expression higher in KTC and TPC (Figure 1C). Therefore, we selected KTC and TPC as experimental cells. Similarly, effective siRNA2 and siRNA3 which considerably reduced the expression of SYT12 in the TPC and KTC cell lines (Figure 1D) for the following experiments. Whereafter, CCK-8 assays 
and cell colony formation assays were achieved. We discovered that knocked down the expression of SYT12 observably suppressed proliferation ability of PTC cell lines (KTC and TPC) (Figure 2).

\section{Knock-down of SYT 12 inhibited the trans-well migration and invasion of PTC cells}

As is mentioned before the higher expression of

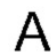

A

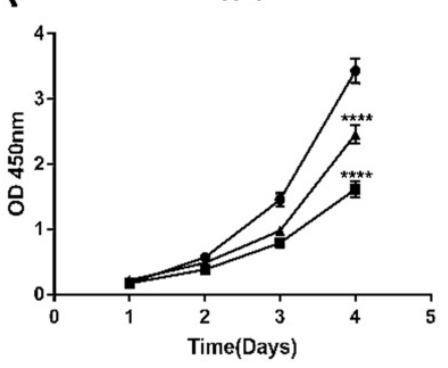

C
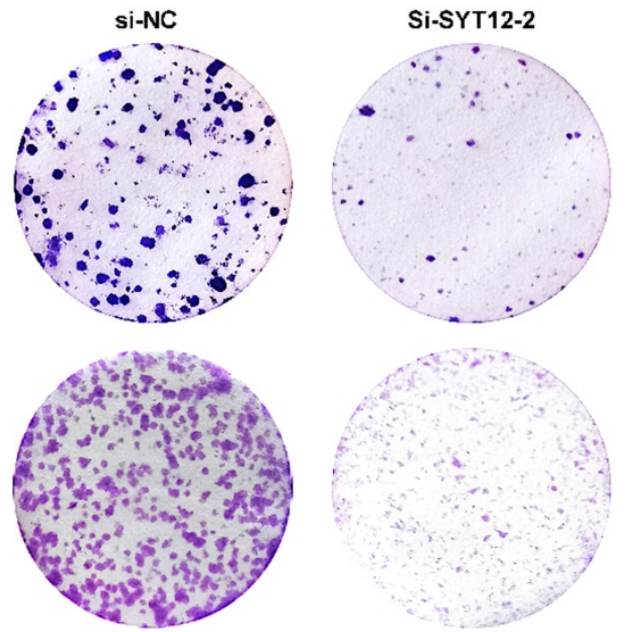

$\rightarrow$ Si-NC

- Si-SYT12-2

- Si-SYT12-3

B

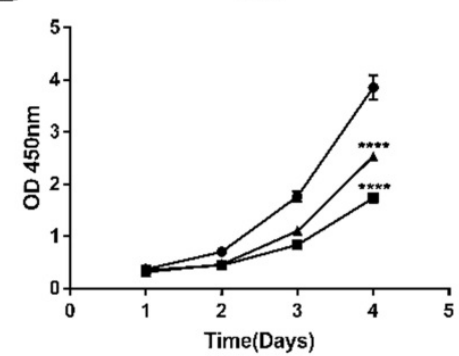

$\rightarrow$ Si-NC

- Si-SYT12-2

$\star$ Si-SYT12-3
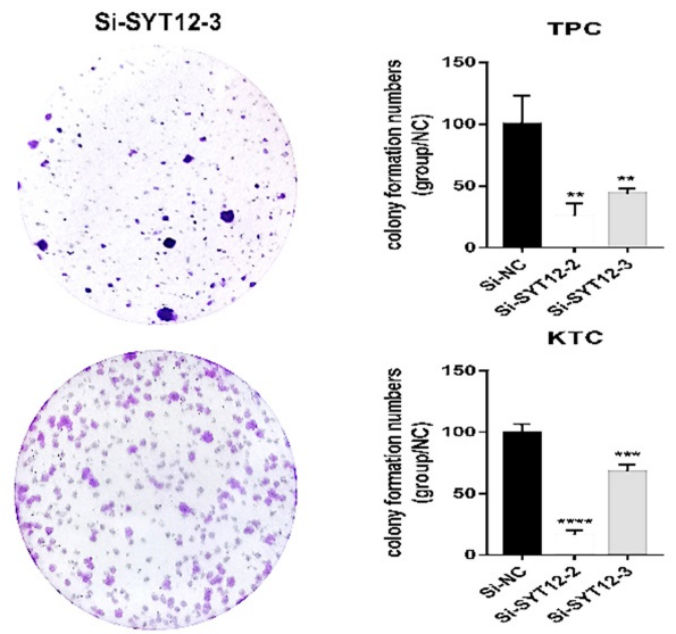

SYT12 was correlated with LNM in the biological information analysis, thus we demonstrated the character of SYT12 in PTC by trans-well migration and trans-well invasion assays which often use to evaluate tumors metastasis capacity. The result of the cell migration assays indicated that number of wandering cells of TPC (Figure 3A \& B) and KTC (Figure 3C \& D) cells which shifted from upper

Figure 2. Down-regulated SYT1 2 expression inhibited TPC and KTC cell proliferation abilities. (A) CCK-8 assays performed in TPC cell lines. (B) CCK-8 assays were performed in KTC cell lines. (C) Colony formation assay in TPC and KTC cells and a corresponding number of colonies. $* \mathrm{P}<0.05$, $* * \mathrm{p}<0.01$, $* * * \mathrm{p}<0.001, * * * * \mathrm{p}<0.0001$.

A

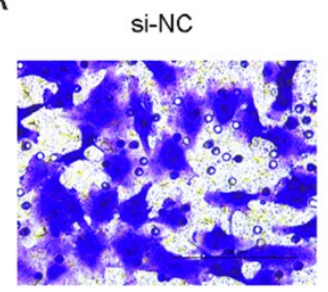

C

si-NC

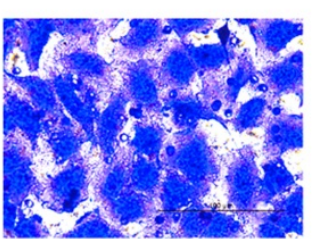

Si-SYT12-2

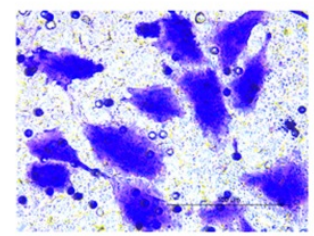

Si-SYT12-2

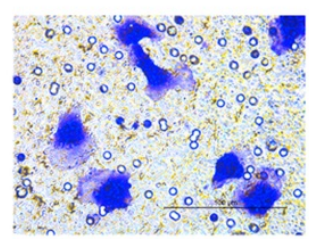

Si-SYT12-3

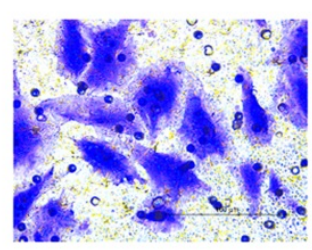

$\mathrm{D}$

Si-SYT12-3

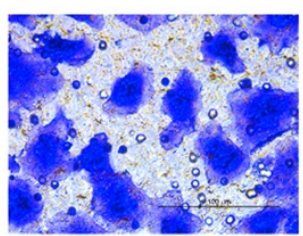

B
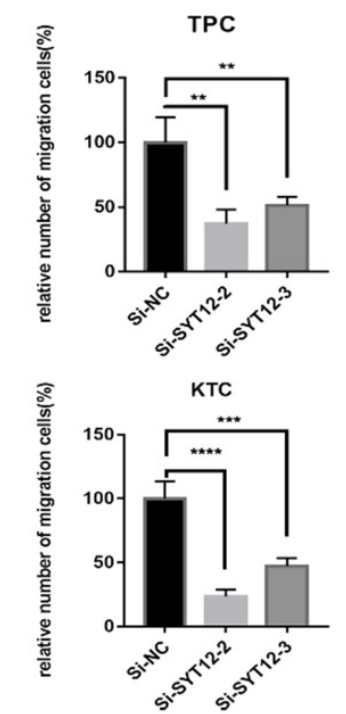

Figure 3. Down-regulation SYT12 gene expression in TPC and KTC cells inhibits migration. (A, B) Transwell migration assays in downregulation SYT12 cells and their corresponding control cells in TPC. (C, D) Transwell migration assays in downregulation SYT12 cells and their corresponding control cells in $\mathrm{KTC} * \mathrm{P}<0.05$, $* * \mathrm{P}<0.01, * * *$ $\mathrm{P}<0.001$, **** $\mathrm{P}<0.0001$. 
chamber to the nether chamber exhibited a different result. The wandering cells which were transfected with si-SYT12s were significantly more than cells transfected with si-NC. The result of the trans-well invasion assays was the same as the migration assay (Figure 4). In a word, knock-down of SYT12 suppressed the TPC and KTC trans-well migration and trans-well invaded ability.

\section{Knock-down SYT 12 promoted the cell apoptosis of PTC cells}

It is well-know that most tumor cells display a property, owing low apoptosis rate. To further probe

A

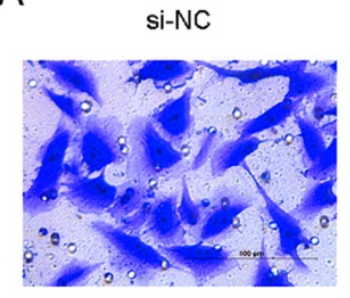

C

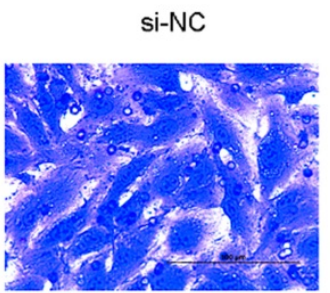

Si-SYT12-2

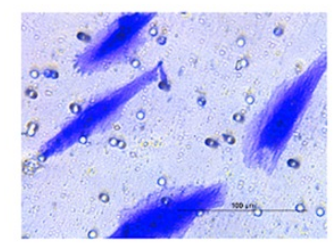

Si-SYT12-2

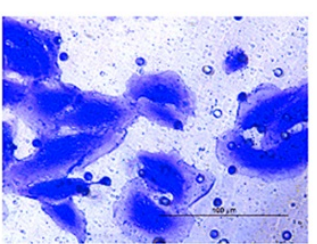

the relationship between SYT12 expression and the tumorigenesis of PTC cells, apoptosis assays were done. We applied flow cytometry to measure cell apoptosis in TPC and KTC cell lines after transfection. Then, we quantified the results by the number of early apoptotic cells plus the late apoptotic cells. We discovered that knock-down SYT12 in KTC and TPC cell lines could promote cell apoptosis, especially in early apoptotic cells (Figure 5). In short, downregulation SYT12 promoted the PTC cell apoptosis capacity.

Figure 4. Down-regulation SYT12 gene expression in TPC and KTC cells inhibits invasion. Transwell invasion assays in downregulation SYT12 cells and their corresponding control cells in TPC. (C, D) Transwell invasion assays in downregulation SYT12 cells and their corresponding control cells in $\mathrm{KTC} * \mathrm{P}^{*}<0.05$, $* * \mathrm{P}<0.01, * * * \mathrm{P}<0.001$, $* * * * P<0.0001$
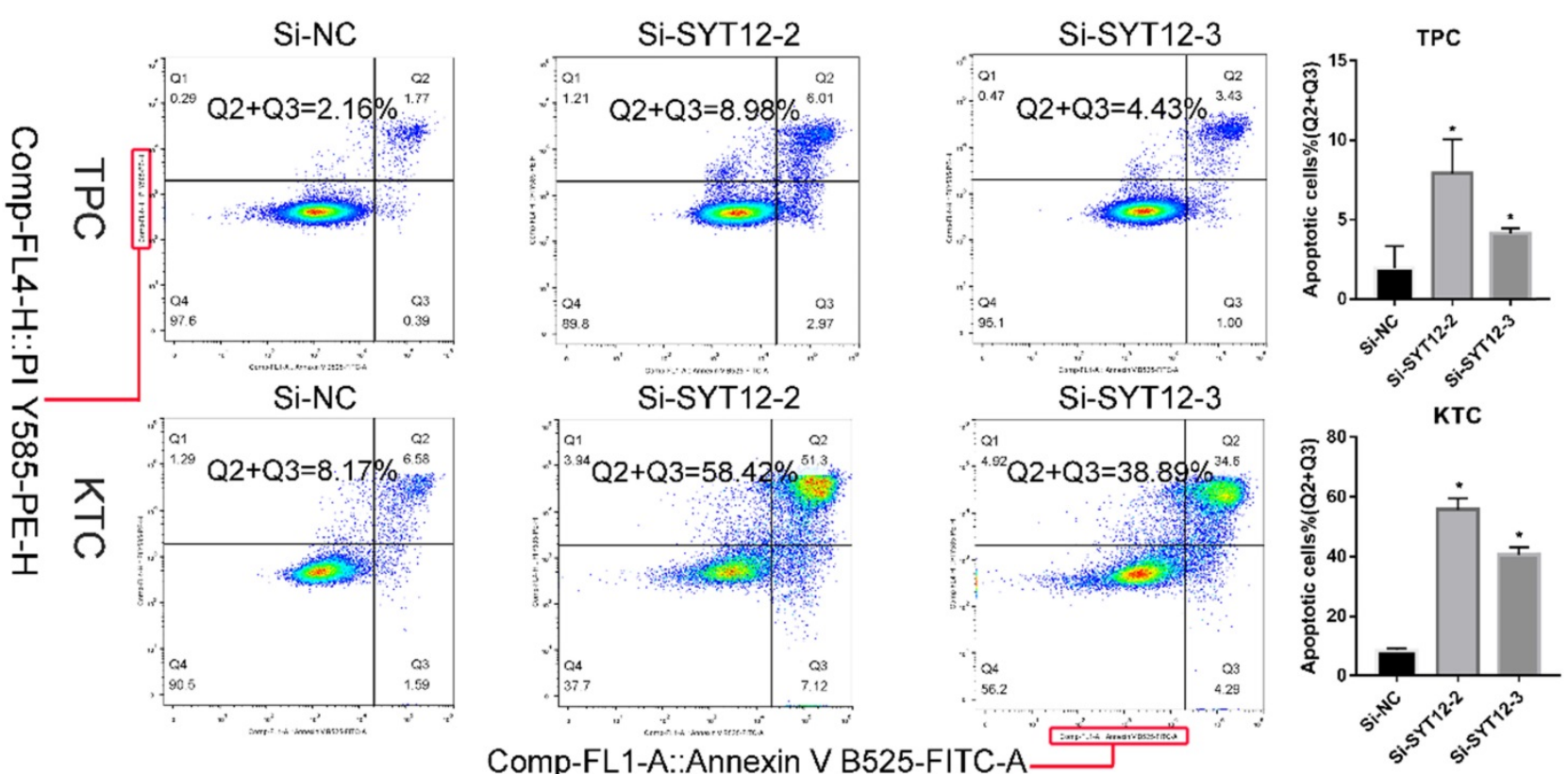

Figure 5. Down-regulation SYT12 gene promote apoptosis TPC and KTC cells. $* \mathrm{P}<0.05, * * \mathrm{P}<0.01, * * * \mathrm{P}<0.001, * * * * \mathrm{P}<0.0001$. 


\section{Discussion}

Thyroid cancer has developed one of the most frequent malignant tumors in current years, and the morbidity number of thyroid malignancies also increased rapidly [13]. At the same time, the patients who got thyroid malignancy were younger on average [2]. Rahib et al. predicted that thyroid cancer would outstrip colorectal cancer by the year 2030 and develop the $4^{\text {th }}$ most frequently identified cancer [14]. But the mortality rate of thyroid cancers has remained stable which is different from the increasing incidence of thyroid cancer in the same period [4-6]. That is to say, the thyroid may be over-diagnosed at present. Furthermore, Bray et al. showed research that there was an estimated 50\%-90\% overdiagnosis rate for thyroid cancer with the influence of regions and health care environment [4]. Although most patients with thyroid cancer had a good prognosis, there still were some thyroid cancer patients with local invasion or distant metastasis who more likely to appear recurrence or death [15]. The overall survival rate of patients with partial recrudescence was about $70 \%$ to $85 \%$, and the overall survival rate of patients with distant metastasis was $30 \%$ to $60 \%[16,17]$. At present, surgeons make treatment decisions mainly relying upon the pathologic parameters and present clinical, which are inadequate to distinguish therapy and estimate the risk for different PTC patient [12]. So, it is crucial to find a more effective way to differentiate low-risk thyroid malignant tumor patients and high-risk thyroid malignant tumor patients.

Thyroid malignancy is a greatly multifarious disease which can be divided into PTC, FTC, primary thyroid lymphoma, medullary thyroid cancer, anaplastic thyroid cancer and primary thyroid sarcoma [3]. PTC is the most familiar kind of thyroid malignancy and its tumorigenesis is a very complex process [18]. According to numerous previous studies, we realized that the tumorigenesis and progression of thyroid malignancy were mostly actuated by genomic mutation, comprising the silencing of contra-oncogenes and initiation of cancerpromoting genes $[7,19,20]$. Between these genes, BRAF V600E was the very well-known [21, 22]. However, the single BRAF V600E was not enough to predict PTC patients' prognosis [23, 24]. These provided a new idea that if we could find novel molecular biomarkers combined with BRAF V600E, we might get a more efficient way to predict the risk of PTCs in clinical practice.

To discover a gene that associates with PTC that could differentiate low-risk and high-risk PTC patients, we send 39 paired PTC samples for RNA sequencing and discovered that SYT12 was overexpressed in PTC tissues associated with nearby non-neoplastic thyroid tissues. There are a few reports about SYT12, and it has been found that played a crucial role in some cancers such as oral cancer and lung adenocarcinoma [10, 11]. Jonklaas et al. also showed that SYT12 could predict papillary thyroid cancer outcomes in a prospective cohort [12]. But the part of the SYT12 gene taking in occurrence and development of thyroid tumor has yet remained unclear.

In the present analysis, we identified that SYT12 was obviously overexpressed in PTC tissues associated with normal tissues in the TCGA cohort according to the bioinformatics analysis. And it was consistent with the RT-qPCR analysis of collected 40 paired surgical tissue samples and thyroid cell lines. Also, it was shown that higher expression of SYT12 was a risk factor for LNM and increased the incidence rate of primary neoplasm multivariate focus type and classical histological type in patients with PTC by logistic regression in the TCGA cohort.

Loss-of-function assays presented that knocked down SYT12 could inhibit the proliferation, migration, colony formation, and invasion abilities of PTC cells in vitro. Furthermore, SYT12 knock-down could increase the apoptotic rate of PTC cells. These results were reliable with clinicopathological descriptions that SYT12 was related with LNM, primary neoplasm focus type, and histological type. In brief, SYT12 is an oncogene that promotes thyroid carcinoma progression and metastasis in PTC.

Nevertheless, our research has some limitations. Initially, the quantity of local samples is too small, and more cases are required to provide more strict results. Secondly, our assays were all in vitro and SYT12 function should be confirmed in vivo by additional animal model assays. Thirdly, the mechanism of SYT12 in PTC's occurrence and development yet remain unclear and compels additional study. Lastly, data of disease-free survival and overall survival lacked, thorough data collection is necessary for discussing the prognostic value of SYT12.

As a whole, our results indicate that SYT12 was up-regulation in primary PTC and its downregulation could inhibit proliferation, migration, invasion, and facilitates cell apoptosis. This study showed that SYT12 was a probable biomarker for diagnosis and prognosis in PTC.

\section{Acknowledgements}

This work was supported by Wenzhou Science and Technology Planning Project (Y20190204). The authors would like to thank all the doctors of the Department of Breast Surgery, The First Affiliated Hospital of Wenzhou Medical University, (Wenzhou, 
China) for providing all the necessary information required for this study.

\section{Ethical Approval and Consent to participate}

Ethical approval for this study was obtained from the Ethics Committee of the First Affiliated Hospital of Wenzhou Medical University.

\section{Funding}

Natural Science Foundation of Zhejiang Province, Grant/Award Number: LY18H160053; Science and Technology Project of Wenzhou, Grant/ Award Number: Y20170030.

\section{Consent for publication}

Written informed consent was issued by the patients for the publication of this research and accompanying images. A copy of the written consent is ready for review by the Editor in Chief of this journal.

\section{Availability of data and materials}

The data sets supporting the conclusions of this study are included in this article and its, additional images. Raw data are available on the main electronic data storage system of First Affiliated Hospital of Wenzhou Medical University and access can be provided upon request to the authors.

\section{Competing Interests}

The authors have declared that no competing interest exists.

\section{References}

[1] Siegel RL, et al. Cancer statistics, 2020. CA Cancer J Clin 2020;70(1): 7-30.

[2] Miller KD, et al. Cancer statistics for adolescents and young adults, 2020. CA Cancer J Clin 2020;70(6):443-459.

[3] Haugen BR, et al. 2015 American Thyroid Association Management Guidelines for Adult Patients with Thyroid Nodules and Differentiated Thyroid Cancer: The American Thyroid Association Guidelines Task Force on Thyroid Nodules and Differentiated Thyroid Cancer. Thyroid 2016;26(1)

[4] Bray F, et al. Global cancer statistics 2018: GLOBOCAN estimates of incidence and mortality worldwide for 36 cancers in 185 countries. CA: a cancer journal for clinicians 2018;68(6):394-424.

[5] Hong S, et al. Lee, Cancer Statistics in Korea: Incidence, Mortality, Survival, and Prevalence in 2017. Cancer Res Treat 2020;52(2): 335-350.

[6] Chen W, et al. Cancer statistics in China, 2015. CA: a cancer journal for clinicians 2016;66(2):115-132.

[7] Prete A, et al. Update on Fundamental Mechanisms of Thyroid Cancer. Front Endocrinol (Lausanne) 2020;11:102

[8] Brose N, et al. Synaptotagmin: a calcium sensor on the synaptic vesicle surface. Science 1992;256(5059):1021-1025.

[9] Pang ZP, Südhof TC. Cell biology of Ca2+-triggered exocytosis. Curr Opin Cell Biol 2010;22(4):496-505.

[10] Eizuka K, et al. SYT12 plays a critical role in oral cancer and may be a novel therapeutic target. J Cancer 2019:10(20):4913-4920.

[11] Liu K, et al. Shen, Synaptotagmin 12 (SYT12) Gene Expression Promotes Cell Proliferation and Progression of Lung Adenocarcinoma and Involves the Phosphoinositide 3-Kinase (PI3K)/AKT/Mammalian Target of Rapamycin (mTOR) Pathway. Med Sci Monit 2020;26: e920351.

[12] Jonklaas J, et al. Loh, Novel biomarker may contribute to predicting papillary thyroid cancer outcomes. Future Sci OA 2018;4(1): FSO249.

[13] Torre LA, et al. Global Cancer Incidence and Mortality Rates and Trends--An Update. Cancer Epidemiol Biomarkers Prev 2016;25(1):16-27.

[14] Rahib L, et al. Projecting cancer incidence and deaths to 2030: the unexpected burden of thyroid, liver, and pancreas cancers in the United States. Cancer Res 2014; 74(11):2913-2921.
[15] Carling T, Udelsman R. Thyroid cancer. Annu Rev Med 2014;65:125-137.

[16] Sampson E, et al. Clinical management and outcome of papillary and follicular (differentiated) thyroid cancer presenting with distant metastasis at diagnosis, Cancer 2007; 110(7):1451-1456.

[17] Lin JD, et al. Clinical characteristics of poorly differentiated thyroid carcinomas compared with those of classical papillary thyroid carcinomas. Clin Endocrinol (Oxf) 2007;66(2):224-228.

[18] Lloyd RV, et al. Papillary thyroid carcinoma variants. Head Neck Pathol 2011;5(1):51-56.

[19] Xing M. Genetic alterations in the phosphatidylinositol-3 kinase/Akt pathway in thyroid cancer. Thyroid 2010;20(7):697-706.

[20] Liu X, et al. Highly prevalent TERT promoter mutations in aggressive thyroid cancers. Endocr Relat Cancer 2013;20(4):603-610.

[21] Xing M. BRAF V600E mutation and papillary thyroid cancer. JAMA 2013;310(5):535.

[22] Abubaker J, et al. Clinicopathological analysis of papillary thyroid cancer with PIK3CA alterations in a Middle Eastern population. J Clin Endocrinol Metab 2008;93(2):611-618

[23] Nam JK, et al. Is the BRAF(V600E) mutation useful as a predictor of preoperative risk in papillary thyroid cancer? Am J Surg 2012;203(4):436-441.

[24] Ito $Y$, et al. BRAF mutation in papillary thyroid carcinoma in a Japanese population: its lack of correlation with high-risk clinicopathological features and disease-free survival of patients. Endocr J 2009;56(1):89-97. 